\title{
Localized Silicon Fusion and Eutectic Bonding for MEMS Fabrication and Packaging
}

\author{
Y. T. Cheng, Liwei Lin*, and Khalil Najafi \\ Department of Electrical Engineering and Computer Science. \\ *Department of Mechanical Engineering and Applied Mechanics, \\ The University of Michigan, Ann Arbor, MI. 48109-2122, USA. \\ E-mail: ytcheng@engin.umich.edu
}

\begin{abstract}
Localized silicon fusion and eutectic bonding processes based on the technique of localized heating are demonstrated and reported for the first time. Phosphorus-doped polysilicon is applied in the localized, silicon-to-glass fusion bonding experiments. Gold is used in the silicon-to-gold eutectic bonding tests. Polysilicon and gold films are patterned as line-shape resistive heaters and they react as the bonding materials. It is found that both processes can be accomplished in 5 minutes and the high temperature bonding region can be confined in a small area. This new class of bonding scheme has potential applications for MEMS fabrication and packaging that require low temperature processing at the wafer level, excellent bonding strength and hermetic sealing characteristics.
\end{abstract}

\section{INTRODUCTION}

Bonding techniques, including fusion, eutectic, and anodic bonding, have been used in IC and MEMS manufacturing for many years [1,2]. Although still an emerging technology, silicon bonding is already producing such commercial devices as pressure sensors and accelerometers. All of these bonding processes require two basic elements. First, two bonding surfaces must be flat to have intimate contact for bonding. Second, proper processing temperatures are required to provide the bonding energy. For example, the conventional silicon-to-silicon fusion bonding process occurs at a bonding temperature of above $1000^{\circ} \mathrm{C}$ [3]. Anodic bonding, on the other hand, is performed at a much lower temperature of about $450^{\circ} \mathrm{C}$ with the aid of a high electrostatic field [4]. The silicon-gold eutectic state occurs at a temperature of $363^{\circ} \mathrm{C}$ that is the theoretical bonding temperature [5]. Among these bonding processes, one common drawback is the high temperature requirement that may damage and degrade temperature-sensitive materials and integrated circuits. Therefore, they are not generally applicable in fabrication or packaging devices when temperature-sensitive materials exist. For the past few years, many efforts have been undertaken to find a reliable bonding process that can be conducted at a low temperature. Unfortunately, these new bonding processes depend highly on the bonding material [6], surface treatment [7], and surface flatness [8].

This paper presents a new bonding process based on the concept of localized heating. High temperature, localized bonding processes are performed while the whole wafer is maintained at a low temperature. Moreover, localized high temperature is able to cause softening of the bonding material and alleviate the surface roughness problem. Two types of localized bonding processes have been investigated: (1) silicon-to-glass fusion bonding, and (2) silicon-to-gold eutectic bonding. Technical issues in bonding conditions, strength and procedures for both fusion and eutectic bonding are discussed. It is our belief that this technique can greatly simplify MEMS fabrication and packaging at both the wafer and chip levels.

\section{PRINCIPLE OF LOCALIZED HEATING AND BONDING}

Figure 1 shows the experimental setup for the bonding tests: (a) cross sectional view, (b) the design of microheater and (c) the design of a temperature sensor. Figure l(a) shows the cross sectional view where a silicon or glass cap is to be bonded to the device substrate. A silicon dioxide layer is grown on the device substrate for electrical and thermal insulation. In the fusion bonding experiments, polysilicon is grown and patterned as the heating and bonding material. In the eutectic bonding experiment, gold resistive heaters are used as the heating and bonding materials. A proper pressure (about $1 \mathrm{MPa}$ ) is then applied to put the cap substrate in contact with the bonding resistors as shown in Fig. 1(a). Figure 1 (b) shows the design of the enclosed-shape microheater that can be used to encapsulate MEMS devices. In order to measure the temperature surrounding the microheater, a temperature sensor made of polysilicon or gold is placed $15 \mu \mathrm{m}$ away from the bonding area as shown in Fig. 1(c). The temperature is characterized by monitoring the change in resistance

Pressure
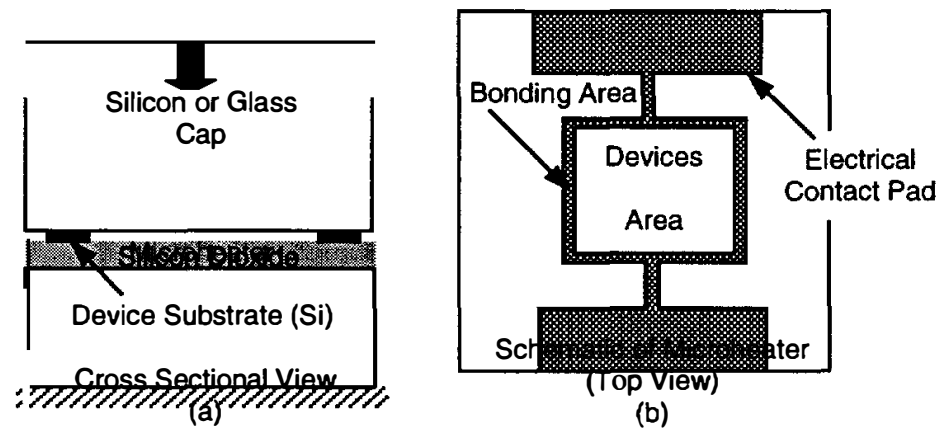

(b)

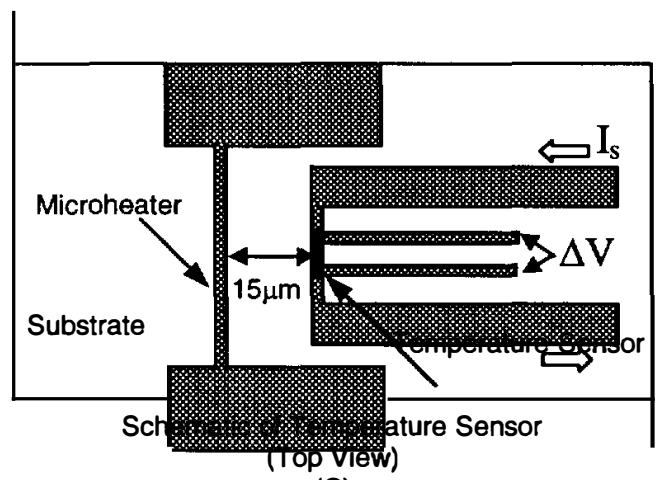

(C)

FIG 1. The schematic of the localized bonding experiments. (a) cross sectional view, (b) schematic of microheater, (c) a temperature sensor. 


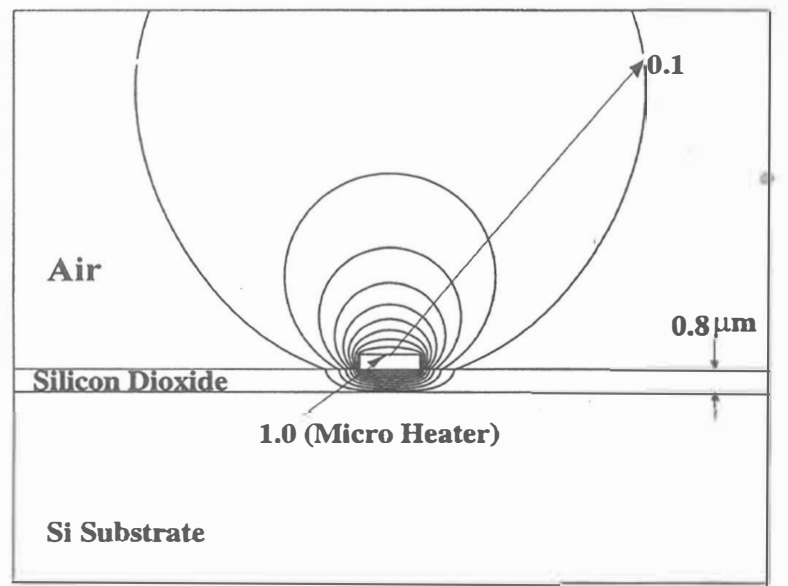

FIG 2. Isotherms around a $2 \mu \mathrm{m}$ wide microheater [9].

Which can be achieved by dividing $\Delta \mathrm{V}$ by the input current $\mathrm{I}_{\mathrm{a}}$. In a recent heat transfer study [9], high temperature region is found to be confined in a very small region. Figure 2 shows the isotherm of such a system. If the microheater is at a high temperature of $1000^{\circ} \mathrm{C}$, the temperature drops to $100^{\circ} \mathrm{C}$ less than $2 \mu \mathrm{m}$ away from the heater as shown. Therefore, localized heating can be achieved by the proper arrangement of microheaters and insulation layers.

Two widths, 5 or $7 \mu \mathrm{m}$, of the microheaters are designed and tested with a square bonding area of $500 \mu \mathrm{m}^{2}$. A pressure of 1 $\mathrm{MPa}$ is applied on top of two wafers and a current, which depends on the design of the microheaters, is passed through the heater to provide the bonding temperature. An electro-thermal model that was previously established based on the conservation of energy [9] was used here to estimate the temperature. Figure 3 shows the simulation results (solid lines) and experiments (symbols) of polysilicon microheaters under different input currents without the cap substrate. The experimental data is calculated by assuming a linear dependence of resistivity with respect to temperature:

$$
\rho(T)=\rho_{o}\left(1+\xi\left(T-T_{o}\right)\right)
$$

where $\rho_{o}$ is the resistivity at room temperature and $\xi$ is the temperature coefficient of resistivity. For N-type polysilicon with dopant concentration of $7.5 \times 10^{19} / \mathrm{cm}^{3}$, this temperature coefficient is about $1.2 \times 10^{-3} /{ }^{\circ} \mathrm{K}[\mathbf{1 0 , 1 1}]$.

The same principle is used in the temperature sensor to determine the temperature changes at a short distance, $15 \mu \mathrm{m}$, away from the microheater. It is found that when an electric current of $30 \mathrm{~mA}$ is passed through the $5 \mu \mathrm{m}$ polysilicon resistive heater, the temperature reaches the melting point of polysilicon $\left(\sim 1415^{\circ} \mathrm{C}\right)$. At the same time, the temperature sensor indicates a temperature increase of less than $40^{\circ} \mathrm{C}$. Apparently, the high temperature region is well confined in a very small region in the device substrate

\section{LOCALIZED FUSION BONDING}

Based on the concept of localized heating, localized siliconto-glass bonding is demonstrated in this paper. First, a Pyrex glass cap substrate (7740 from Dow Corning) is placed and pressed on the top of polysilicon microheaters as shown in Fig. 1(a). A $31 \mathrm{~mA}$ input current which is very close to cause melting of polysilicon is then applied to the $5 \mu \mathrm{m}$ wide, $1.1 \mu \mathrm{m}$ thick polysilicon microheater for about 5 minutes. This input current generates a temperature of about $1300^{\circ} \mathrm{C}$ based on the current-temperature simulation that includes the effect of the glass cap on top of the micro-

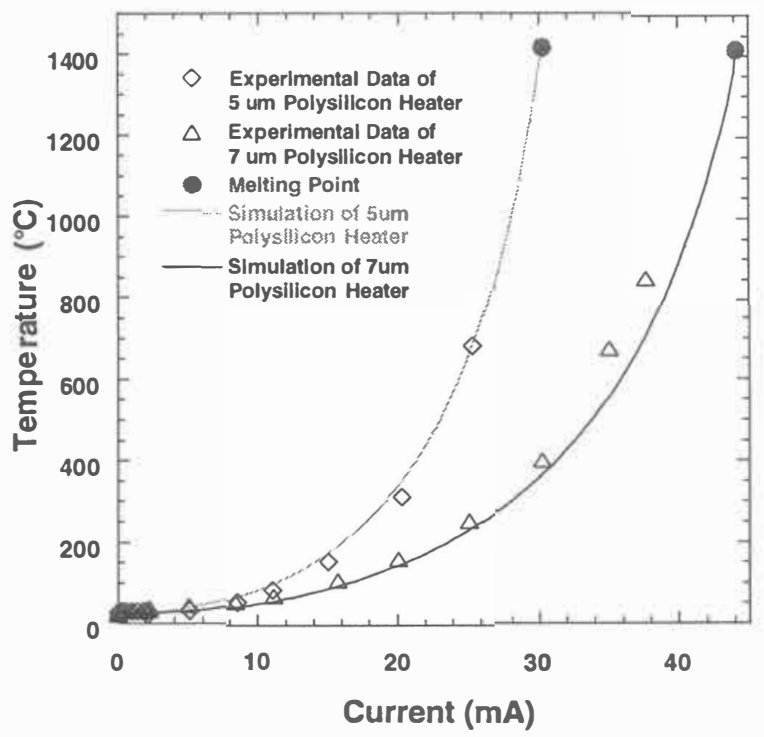

FIG. 3. Experimental and simulation results of 5 and $7 \mu \mathrm{m}$ wide polysilicon microheaters under different current inputs

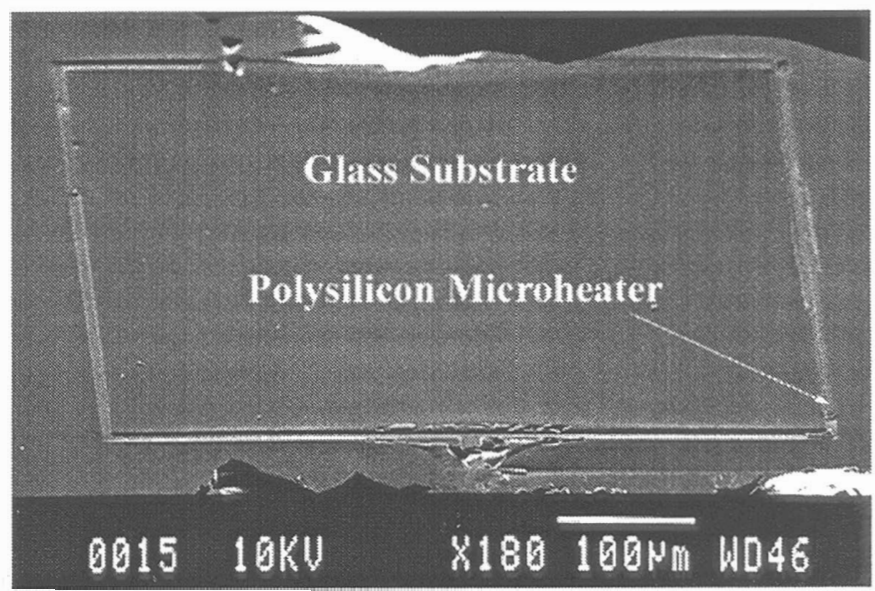

FIG. 4 A SEM micrograph showing the glass cap substrate is softened and has the shape of the polysilicon microheater.

-heater. This temperature is slightly lower than the data shown in Fig. 3 under the same input current because of heat losses to the glass cap.

Figure 4 shows the SEM micrograph of a forcefully broken fusion bond on the glass cap. It is observed that the square shape microheater is reflected on the originally flat glass substrate. Moreover, part of the polysilicon was attached to the glass cap. This microphoto demonstrates two very important features for the localized fusion bonding experiment. First, it is very easy to raise the microheater temperature to be above the glass soften point of $\sim 820^{\circ} \mathrm{C}$ such that the glass cap is locally softened. Second, the applied pressure is high enough to cause intimate contact of the glass cap and the microheaters. Since intimate contact has been made, a good and reliable fusion bond can be expected with proper temperature and reaction time.

In order to determine the bonding strength, a close-up-SEM microphoto was taken as shown in Fig. 5. For this particular sample, the breakage was along one of the microheater. Polysilicon heater, undemeath silicon dioxide layer and the top glass cap can be clearly identified. The morphology of glass near the heater line shows the glass has been softened locally. After dipping the sample into HF solution, the polysilicon heater is clearly 


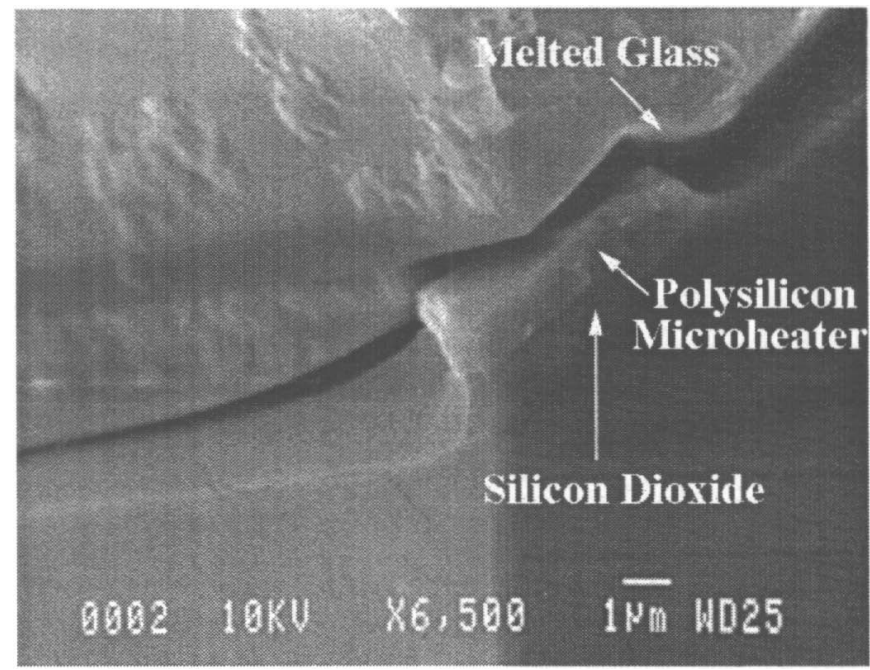

FIG. 5. A SEM micrograph shows the localized silicon-glass fusion bonding. After the bond is forcefully broken, microheater, silicon dioxide and glass cover are clearly observed.

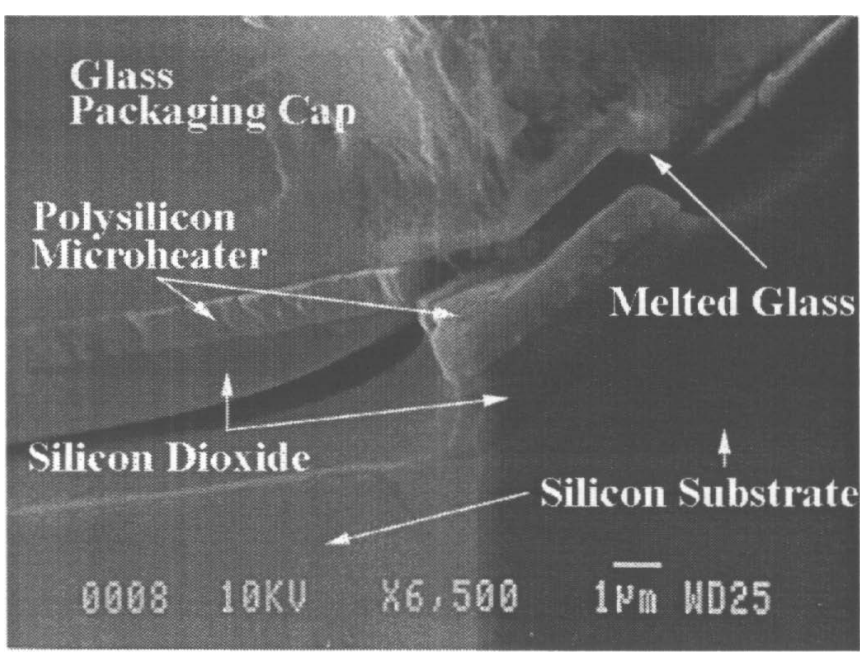

FIG. 6. Polysilicon microheater appears after dipping into HF

delineated as shown in Figure 6. In this case, the polysilicon-glass bond seems to be stronger than the bottom polysilicon-oxide adhesion where the broken trace can be clearly observed. Therefore, these results strongly suggest that an excellent siliconto-glass fusion bonding is achieved.

According to the fusion bonding principle [3], flat surfaces, hydrophilic surface treatment, sufficient high bonding temperature and reasonable bonding time will result in successful bonding. The typical conventional bonding temperature is above $1000^{\circ} \mathrm{C}$ for about 2 hours. In the experiments presented in this paper, microheaters are cleaned by SPM (sulfuric peroxide mixture, i.e., a mixture of $\mathrm{H} \mathrm{SO}_{4}$ and $\mathrm{H}_{2} \mathrm{O}$ clean followed by $\mathrm{HF}$ dip and water rinse for hydrophilic surface treatment [12]. Bright red light emits from microheaters during the bonding process. It is found that when the bonding temperature is raised to very close to the melting temperature of polysilicon, the silicon-to-glass fusion bonding occurs in less than 2 minutes. In another experiment, a lower bonding current of $29 \mathrm{~mA}$ which corresponds to a temperature of about $1000^{\circ} \mathrm{C}$ is applied continuously for 30 minutes. The result shows poor bonding strength and uniformity. According to these experimental results, it is believed that excellent, hermetic bonding

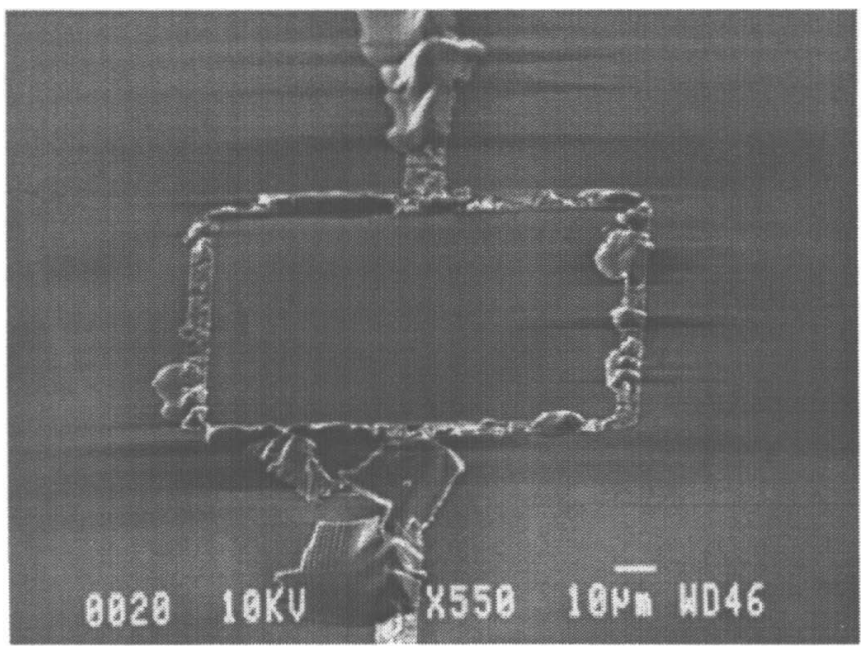

FIG. 7. A SEM micrograph showing localized silicon-to-gold eutectic bonding. After the bond is forcefully broken, silicon is attached to the gold line.

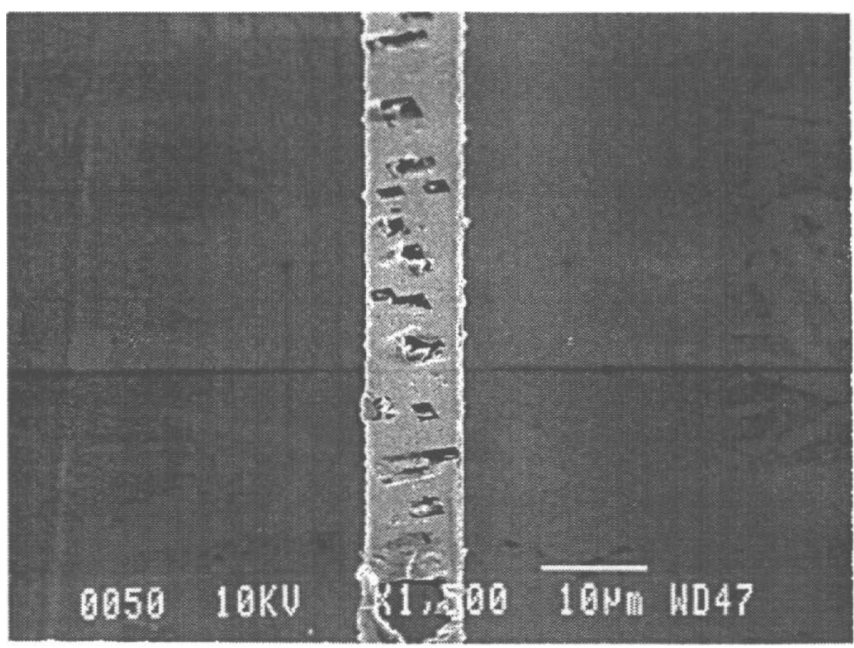

FIG. 8. Non-uniformity is found in a conventional eutectic bonding process.

can be achieved when the bonding temperature is controlled to be very close to the melting point of the polysilion material. At a lower bonding temperature, a longer bonding period is expected to achieve excellent bonding.

\section{LOCALIZED EUTECTIC BONDING}

In the silicon-to-gold eutectic bonding experiments, gold microheaters are used as the heating and bonding materials. A silicon cap substrate is placed on top of the gold microheater as shown in Fig. 1(a). A 0.27 A electric current is then applied to the $5 \mu \mathrm{m}$ wide, $0.5 \mu \mathrm{m}$ thick gold microheater for about 5 minutes. The bonding temperature is estimated to be about $800^{\circ} \mathrm{C}$. During the bonding process, gold diffuses into silicon and the resistivity of gold line increases. It is necessary to increase the current density to maintain a high bonding temperature during the bonding process. Figure 7 shows the result of silicon-to-gold eutectic bonding by the technique of localized heating. It appears that silicon is broken and attached to the gold microheater when the eutectic bond is forcefully broken. Moreover, uniform eutectic bonds can be observed around the square shape microheater. 
For comparison purposes, the same eutectic bonding experiment is also performed by using the conventional eutectic bonding technique $[5,13]$. The process is conducted in an oven that provides global heating and bonding. The processing temperature is first ramped to $410^{\circ} \mathrm{C}$ in 10 minutes and is kept at $410^{\circ} \mathrm{C}$ for 10 minutes before cooling down to room temperature in 10 minutes. Figure 8 shows the bonding result in a SEM microphoto. Nonuniform eutectic bonding can be clearly observed in this photo. This is probably the reason that hermetic sealing was not achieved in a previous report [13]. It is well-known that the diffusivity [14] and solubility [15] of gold into silicon substrate increase when the processing temperature increases. At higher bonding temperatures as those used in the localized bonding process, more gold atoms can diffuse into silicon. Therefore, a thicker layer of gold-silicon alloy can form at a higher bonding temperature [16] and a stronger eutectic bond is expected [17].

\section{DISCUSSION}

Temperature and processing time are the two key factors for both fusion and eutectic bonding if intimate contact has been achieved. In the silicon-to-glass fusion bonding system, atoms obtain thermal energy provided by temperature to overcome reaction barrier to form chemical bonds. In the silicon-to-gold eutectic eutectic

bonding system, diffusion is activated under high temperature environment when atoms overcome the diffusion barrier to from eutectic bonds. It is desirable to have high processing temperatures in both fusion and eutectic bonding processes for higher diffusion constant

and reaction rates. Localized heating provides an excellent way to accomplish the high temperature requirement while maintaining low temperature at the wafer level. Therefore, fast reaction and strong bonding are expected to occur locally. There are many ways to achieve localized heating, including using microheaters or focused micro laser [18]. The key structural design is to prevent the heat losses to the environment or substrate. An insulation layer underneath the heating element serves well for this purpose as illustrated in Fig. 2. Design optimizations can be conducted based on the principle of heat transfer to improve the effectiveness of localized heating.

This paper presents direct silicon fusion and eutectic bonding techniques where the heating elements are also serving as the bonding materials. One drawback for the direct bonding technique is that the bonding materials may diffuse or melt during the process. Therefore, the resistance of the microheater changes and it is very difficult to control the bonding temperature and process. We are currently pursuing the same localized heating idea but adding soldering materials for indirect bonding. Since the microheaters can be preserved during the indirect bonding process, good temperature and process control are expected. Furthermore, encapsulation of microresonators in vacuum environment is underway. We expect to quantitatively analyze the hermetic sealing characteristics of this new technique by monitoring the performance of packaged microresonators.

\section{CONCLUSION}

Localized fusion and eutectic bonding processes have been successfully demonstrated. Phosphorus doped polysilicon and gold resistive heaters are used in silicon-to-glass fusion and silicon-to-gold eutectic bonding processes, respectively. It is found that both processes can be accomplished in less than 5 minutes with excellent bonding strength and uniformity. In the silicon-to-glass fusion bonding process, an input current of about $31 \mathrm{~mA}$ is necessary to reach a bonding temperature of about $1300^{\circ} \mathrm{C}$. In the silicon-to-gold eutectic bonding process, an input current of about $0.27 \mathrm{~A}$ is needed to reach a bonding temperature of about $800^{\circ} \mathrm{C}$. We believe these techniques can greatly simplify MEMS fabrication and packaging at both the wafer and chip levels.

\section{ACKNOWLEDGEMENTS}

The authors would like to thank Prof. Nugyen at the University of Michigan for valuable discussions. Prof. Liwei Lin is supported by the National Science Foundation CAREER award. The project is supported by DARPA.

\section{REFERENCES}

[1] W. H. Ko, J. T. Suminto, and G. J. Yeh, "Bonding Techniques for Microsensors", Micromachining and Micropackaging for Transducers, Elsevier Science Publishers, 1985.

[2] P. W. Barth, "Silicon Fusion for Fabrication of Sensors, Actuators and Microstructures", Sensors and Actuators, A 23, pp. 919 926, 1990.

[3] C. Harendt, H. -G. Graf, B. HÖfflinger, and E. Penteker, J. "Silicon Fusion Bonding and Its Characterization", J. Micromech. Microeng, 2, pp. 113 116, 1992.

[4] G. D. Wallis, and D. I. Pomerantz, "Field Assisted Glass-Metal Sealing", J. Appl. Phys. 40, pp. 3946 3948. 1969

[5] L. Valero, "The Fundamentals of Eutectic Die Attach", Semiconductor Int., 7, pp. 236 241, 1984.

[6] H. Reichl, "Packaging and Interconnection of Sensors", Sensors and Actuators, A, 25, pp. 63 71, 1990.

[7] Q. Y. Tong, and G. Cha, R. Gafiteanu, and U. Gösele, “ Low Temperature Wafer Direct Bonding”, J. MEMS, 3, pp. 29 35.1994.

[8] B. Ziaie, J. Von Arx, M. Dokmeci, and K. Najafi, "A Hermetic Glass-silicon Micropackages with High Density On-Chip feedthroughs for Sensors and Actuators", J. MEMS, 5, pp. 166 179, 1996.

[9] Liwei Lin, "Selective Encapsulations of MEMS: Micro Channels, Needles, Resonators, and Electromechanical Filters", Ph.D. Thesis, UC Berkeley, 21, 1993.

[10] N. C. C. Lu, L. Gerzberg, and J. D. Meindl, " A Quantitative Model of The Effect of Grain Sizeon the resistivity of polycrystalline silicon films", IEEE Electron Device Letter, EDL1, pp. 38 41, 1980.

[11] J. Y. W. Seto, "The Electrical Propertiesof Polycrytalline Silicon” J. Appl. Phys. , 46, pp. 5247 5254, 1975.

[12] K. Ljungberg, Ulf Jansson, S. Bengtsson, and A. Söderbärg, “ Modification of Silicon Surface with $\mathrm{H}_{2} \mathrm{SO}_{4}: \mathrm{H}_{2} \mathrm{O}: \mathrm{HF}$ and $\mathrm{HNO}_{3}: \mathrm{HF}$ for Wafer Bonding Applications", J. Electrochem. Soc., 143, pp.1709 1714, 1996.

[13] M. B. Cohn, Y. Liang, R. Howe, and A. P. Pisano, "Wafer to Wafer Transfer OF Microstructures for Vacuum Package", Technical Digest of the 1996 Solid State Sensor and Actuator Workshop, Hilton Head, pp. 32 35, 1996.

[14] CRC Materias Science and Engineering Handbook, pp. 255, 1997.

[15] H. Okamoto, and T. B. Massalaski, Binary Phase Diagram, pp. $312,1986$.

[16] P. -H, Chang, G. Berman, C. C. Chen, "Transmission electron microscopy of gold-silicon interactions on the backside of wafers", J. Appl. Phys., 63, pp. 1473 1477, 1988.

[17] A. L. Tiensuu, M. Bexwell, J.-Schweitz, L. Smith, and S. Johansson, "Assembling three-diamensional microstructures using gold-silicon eutectic bonding", Sensors and Actuators A , 45, pp. 227 236, 1994.

[18] C. L. Rambin, and R. O. Warrington, "Micro-assembly with A Focused Laser Beam", Proceeding of the IEEE Micro Electro Mechanical System, pp. 285 290, 1994. 